\title{
Tumor miofibroblástico de vejiga
}

\author{
I. Gómez García, R. Molina Burgos, E. Fernández Fernández, A. Palacio España,
} F. González Chamorro, E. Álvarez*, S. Conde Someso**

Servicio de Urología de Adultos y *Servicio de Anatomía Patológica. Hospital San Rafael. **Servicio de Cirugía General. Hospital Ramón y Cajal. Madrid.

Actas Urol Esp 2005; 29 (5): 611-614

\section{ABSTRACT \\ TUMOR MIOFIBROBLASTICO DE VEJIGA}

El tumor miofibroblásticos es un tumor mesenquimal benigno de carácter excepcional, siendo su localización mas habitual es el pulmón; mientras que su aparición en la vejiga, es excepcional, no existiendo mas de 100 casos publicados, de este tipo de tumor en la vejiga. Este tipo de tumor queclínica y radiológicamente, se comporta como un tumor maligno. El diagnóstico patológico es complejo, debido a su similitud con los sarcomas, siendo necesario recurrir a la inmunohistoquímica para un diagnostico de certeza.El tratamiento mediante resección amplia suele ser suficiente no existiendo en la actualidad ningún caso de metástasis a distancia, ni de malignizacion. Presentamos un nuevo caso de este tumor, realizando una amplia revisión bibliográfica.

Palabras clave: Pseudotumor inflamatorio. Pseudosarcoma vesical.

\section{ABSTRACT}

\section{MIOFIBROBLASTIC TUMOR OF BLADDER}

The miofibroblastic tumor, is a mesenchimal benign tumor of exceptional character, being its localization but habitual it is the lung; while its appearance in the bladder, is exceptional, not existing but of 100 published cases, of this tumor type in the bladder. This tumor type that clinic and radiologics, behave as a wicked tumor. The pathological diagnosis is complex, due to its similarity with the sarcomas, being necessary to appeal to the inmunohistoquimics for a I diagnose of certainty. The treatment by means of wide resection is usually enough not existing any case of metastasis at the present time at distance, neither of malignization. We present a new case of this neoplasm, carrying out a wide bibliographical revision.

Keywords: Inflammatory pseudotumor. Bladder pseudosarcoma.

Más del 95\% de los tumores vesicales son de origen urotelial, los restantes suelen ser tumores de origen mesenquimal, siendo el mas frecuente el leiomioma. El tumor miofibroblastico (TM) o pseudotumor inflamatorio es un tumor benigno e infrecuente, que asemeja a los sarcomas vesicales, siendo solo posible su distinción mediante el estudio patológico e inmunohistoquímico.

\section{CASO CLINICO}

Varón de 46 años con síndrome miccional y urgencia de 4 meses de evolución, acompañado de hematuria total con coágulos. Refiere frecuencia miccional de dos horas y nicturia de dos ocasiones. Presenta leve síndrome obstructivo, con tenesmo y estranguria. No episodios de infección de orina ni de cólico nefrítico. No síndrome constitucional. Como antecedentes de interés, destacan fumador de dos paquetes al día y vasectomizado.

La exploración física es anodina. Pruebas complementarias: Hemograma destaca hemoglobina de 10,2 g/dl; bioquímica y estudio de hemostasia normales. PSA 0,32. Ecografía: riñones 
normales sin alteraciones en el parénquima ni en el seno; vejiga tumor de aspecto sólido en fondo y cúpula vesical de 5 centímetros de diámetro máximo (Fig. 1).

Con el diagnóstico de tumor vesical se realiza resección transuretral de tumor vesical y base de implantación, de aspecto sólido localizado en fondo y cúpula vesical. El estudio patológico informa de proliferación fusiforme con moderada atipia celular y escasas mitosis (Fig. 2), que se ordenan formando fascículos entre los que se observan sustancia fundamental y fibras conjuntivas. Las técnicas inmunhohistoquímica demuestran positividad para vimentina, actina y ALK (Figs. 3, 4 y 5); marcando en menos del 5\% para Ki67; siendo negativas para desmina, proteína S-100 y pancitoqueratina; siendo todos estos hallazgos sugestivos de tumor miofibroblastico de bajo grado.

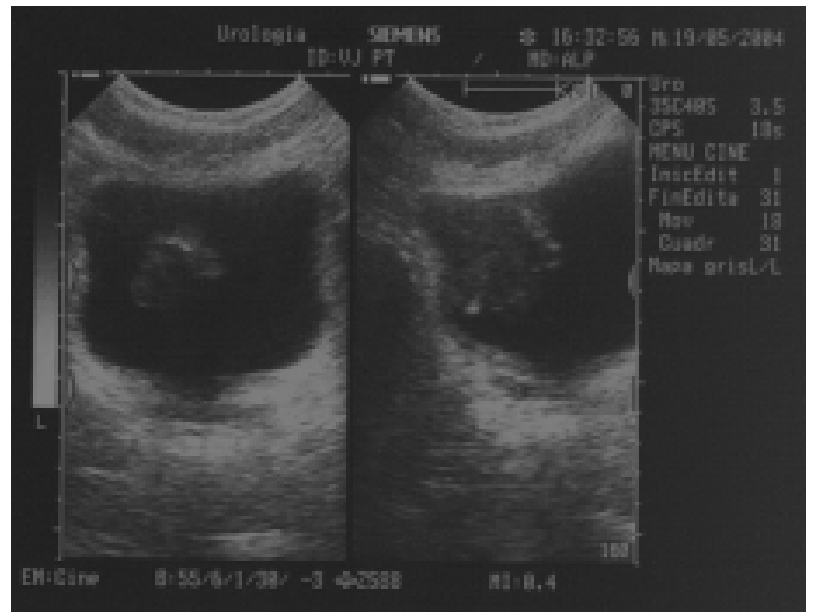

FIGURA 1. Ecografia: Tumor vesical localizado en cúpula.

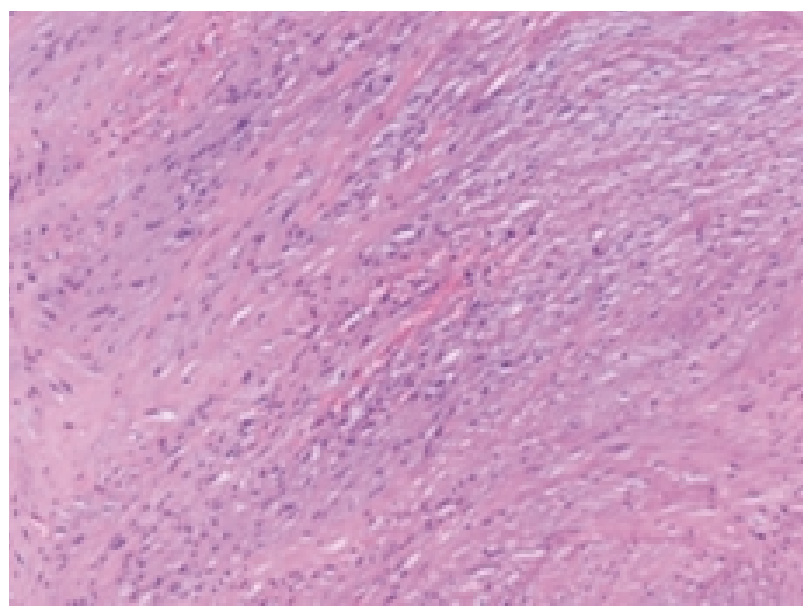

FIGURA 2. Hematosilina eosina.

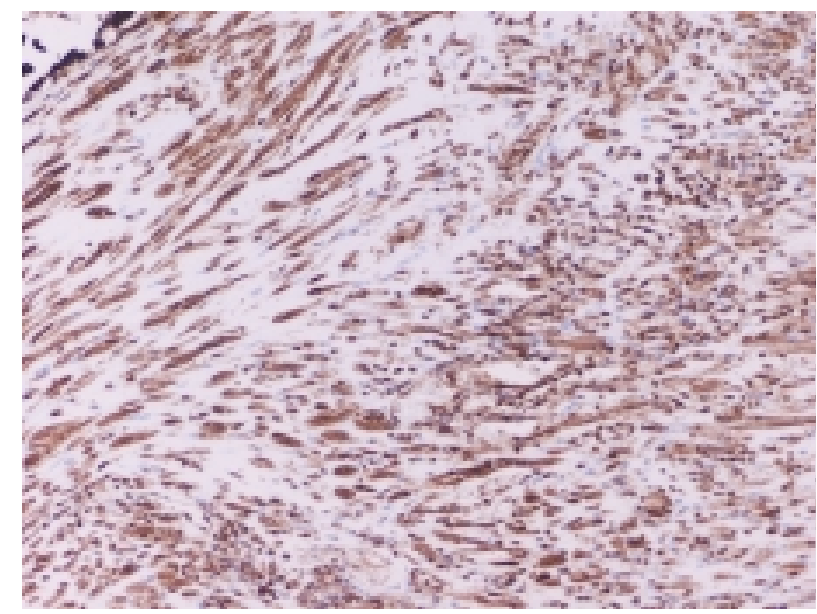

FIGURA 3. Tinción de Vicentina positiva.

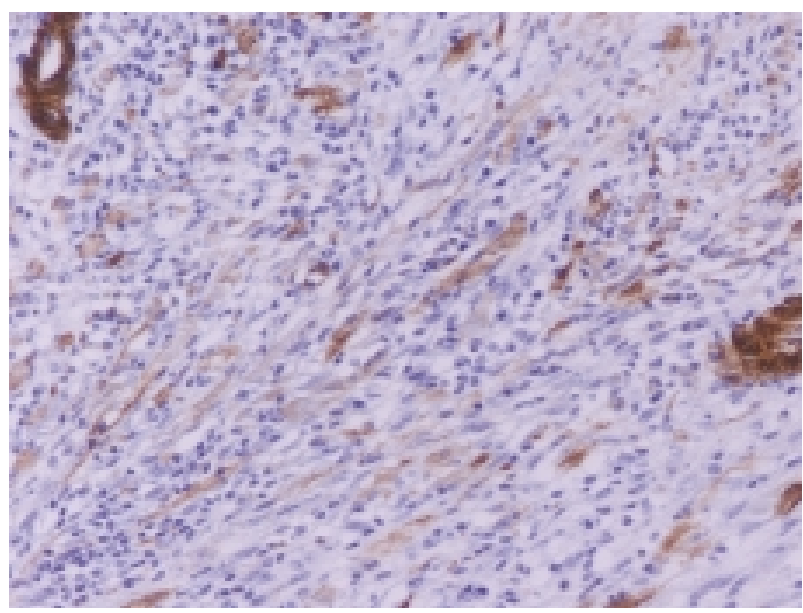

FIGURA 4. Tincion de Actina positiva.

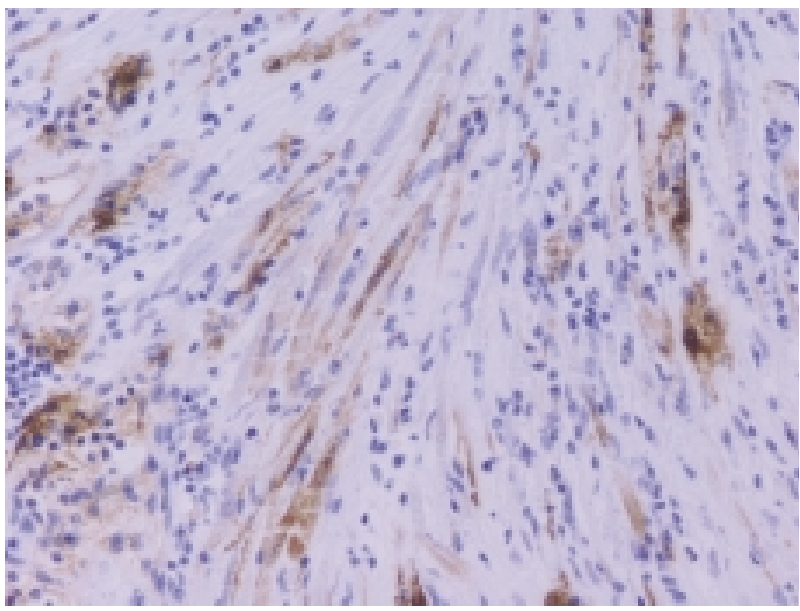

FIGURA 5. Tinción de ALK positiva.

Seis meses de la resección se realiza exploración vesical bajo anestesia con toma de biopsia sobre área de resección previa, siendo informado el estudio patológico como tejido urotelial normal. 


\section{DISCUSIÓN}

El pseudotumor inflamatorio, o tumor miofibroblastico fue descrito por primera vez en el hígado, en 1980 Roth informa del primer caso de afectación vesical $^{1}$. El tumor miofibroblastico ha recibido distintos nombres, pseudotumor inflamatorio, pseudosarcoma fibromixoide, fascitis nodular, pseudosarcoma, proliferación miofibroblastica pseudosarcomatosa, nódulo postoperatorio de células de huso. Este inusual tumor ha sido descrito, en pulmón, hígado, y tracto genitourinario $^{2}$. En el aparato genitourinario, su localización mas común es la vejiga, aunque están descritos casos de localización uretral, prostática y de pelvis renal ${ }^{3}$. La localización vesical es infrecuente, con no mas de 90 casos descritos en la literatura, dada su similitud clínica e histológica con el leiomiosarcoma vesical, es imprescindible el correcto estudio patológico e inmunohistoquimico. El TM se considera una lesión benigna en la que existe una respuesta inflamatoria anómala y excesiva, simulando a los sarcomas.

No parece que exista diferencia entre ambos sexos $^{4}$, pudiéndonos encontrar casos descritos desde los dos meses de edad hasta los 87 años $^{3-}$ ${ }^{9}$ siendo mas habitual su aparición en la tercera década de la vida. Existen autores que defienden su mayor frecuencia en el sexo femenino ${ }^{5,6,8,9}$.

El pseudotumor inflamatorio vesical es de etología desconocida, aunque existen diversas teorías: secundario a factores irritativos crónicos ${ }^{4,5}$, cirugías vesicales previas ${ }^{4,10}$, histerectomía, cistitis, cateterismos ureterales, vejiga neurógena y resecciones vesicales previas ${ }^{6,11}$, antecedentes traumáticos, infecciones urinarias o consumo de drogas ${ }^{4}$.

El síntoma princeps es la hematuria total, y

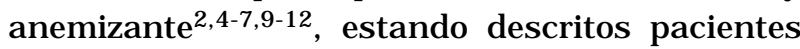
con urgencia miccional como único síntoma ${ }^{8,13}$. Existen casos en los que la hematuria se acompaña de clínica constitucional (fiebre, anemia, perdida de peso $)^{3}$. La sintomatología sistémica se explica por la sobre expresión del factor de necrosis tumoral alfa (TNFa) ${ }^{3}$.

Radiológicamente se aprecia lesiones tumorales grandes y exofiticas, soliendo afectar a cualquier área de la vejiga, pero respetando habitualmente el trígono ${ }^{4,5,9}$. Endoscópicamente se aprecia lesión sólida, exofítica, ulcerada y nodular; se han descritos casos aislados en los que no existen lesiones excrecentes ${ }^{1,4,5}$.
El diagnóstico patológico es difícil, debido a su rareza, y la simulación con los sarcomas vesicales. Macroscopicamente son tumores de tamaño variable desde los dos, hasta los 11 centímetros ${ }^{1,4,7,12-15}$. Histológicamente son tumores de origen mesenquimal que tienen características patológicas similares a la fascitis nodular ${ }^{14}$.Esta compuesto por células fusiformes (células elongadas) que microscópica e inmunohistoquimicamente asemejan a miofibroblastos ${ }^{5}$.Las células fusiformes se agrupan formando husos, en un estroma de características mixoides y edematosa lleno de células inflamatorias y pequeños vasos sanguíneos ${ }^{1}$; ocasionalmente se pueden apreciar glóbulos rojos. Las células musculares se caracterizan por un citoplasma intensamente eosinofílico. Los núcleos celulares son alargados y grandes, con nucleolos prominentes no evidenciándose atípia, apreciar mitosis intranucleolar ${ }^{12}$. Entre las células musculares se puede apreciar infiltrado inflamatorio que abarca linfocitos, neutrófilos y eosinófilos ${ }^{5}$. La lesión esta confinada a la lamina propia, pudiendo afectar a la musculares mucosa. Las células fusiformes pueden confundirnos con células mesenquimales malignas, en especial con células musculares lisas ${ }^{1}$. El estroma en el pseudotumor inflamatorio suele presentar características mixoides e intenso edema. Tamaño pequeño de la lesión, homogeneidad celular, y la ausencia de alteaciones mitóticas van a favor del pseudotumor inflamatorio ${ }^{5}$; la existencia de necrosis ${ }^{6}$ y la determinación de proteínas de células musculares lisas mediante estudios inmunohistoquimicos y ultraestructurales, abogan por el diagnostico de leiomiosarcoma, mientras que la evidencia de células miofibroblásticas orientan el diagnostico hacia el pseudotumor inflamatorio ${ }^{5,10}$.

El TM, inmunohistoquimicamente presentan características similares al fibroblasto y miofibroblasto, aunque se ha descrito un paciente con alteración en la expresión de cytoqueratina ${ }^{12}$. La reactividad a vimmentina es la norma, siendo también postiva en la mayor parte de los casos a la actina y al ALK. La positividad de las diferentes técnica inmunohistoquimicas a proteínas de célula de músculo liso, como la desmina y miogenina (MYCF-4, Myo-d) orienta hacia el diagnóstico de sarcoma. La determinación desmina según 
algunos autores es inespecífica ya que puede ser expresado tanto por los sarcomas como por los $\mathrm{TM}^{6,12}$. Los estudios citogénicos y el análisis del cariotipo demuestra que en los tumores benignos vesicales existe una alteración en el brazo corto del cromosoma 12 en concreto en los sectores q13 y q15, hecho que nos puede orientar hacia el diagnostico de $\mathrm{TM}^{16}$. Existen pacientes en los que se ha demostrado la presencia de $\mathrm{ADN}$ viral ${ }^{16}$, siendo descrito también la presencia de inclusiones intracitoplasmaticas que asemejan a la Chlamydia ${ }^{8}$.

El diagnostico diferencial debe realizarse con el leiomiosarcoma vesical en adultos, rabdomiosarcoma en los niños, tumor sarcomatoide vesical, y la cistitis intersticial ulcerosa ${ }^{2,4,10,12}$. El infiltrado inflamatorio es mas pronunciado y mas heterogeneo que en el leiomiosarcoma, en el cual suele existir solo un tipo de célula inflamatoria.

Se han tratado pacientes con reseccion transuretral encontrándose libres de enfermedad a al año de la intervención ${ }^{6,7,10}$. También se ha sometido a pacientes a cistectomía parcial estando libres de enfermedad a los 4 años de la intervención ${ }^{2,4,5,8,14}$. En los casos encontrados en la literatura no se han encontrado casos de recidiva ni de malignización. No existen casos en la literatura de metástasis a distancia, aunque si de recurrencia a nivel local ${ }^{6}$. Debido a que constituye un tumor de naturaleza benigna la actitud conservadora es la norma, aunque debido su similitud con las sarcomas vesicales se recomiendo un seguimiento estricto.

\section{REFERENCIAS}

1. Roth JA. Reactive pseudosarcomatous response in urinary bladder. Urology 1980;16:635.

2. Zubac ZP, Malmfred S, Nesrtrom B. Inflammatory pseudotumor of the bladder. Scans J Urol Nephrol 2000;34:72-74.

3. LakShmanan Y, Wills ML, Gearhart JP. Inflammatory (pseudosarcomatous) myofibroblastic tumor of the bladder. Urology 1997;50:285-288.

4. Stark GL, Feddersen R, Lowe BA, et al. Inflammatory Pseudotumor (Pseudosarcoma) of the bladder. J Urol 1989;141: 610-612.
5. Coyne JD, Wilson G, Shandu D, Young RH. Inflammatory pseudotumor of the urinary bladder. Histophatology 1991; 18:261-264.

6. Iczkowski K. A., Shanks J.H., Gadaleanu V., et al: Inflamatory pseudotumor ans sarcoma of urinary bladder: differential diagnosis and outcome in thirty eight sindle cell neoplasms. Mod Pathol 2001;14:1043-1051.

7. Abascal Junquera JM, Trilla Herrera E, Esquena Fernández S, et al. Pseudotumor inflamatorio vesical. Actas Urol Esp 2004;28:62-64.

8. Gyftopoulus K, Serafetinidis E, Sambaziotis D, et al. Pseudomalignant cell proliferation of the urinary bladder: an unusual cause of voiding symptoms in a young patients. Urology 2002;60:698.

9. Arocena Lanz F, Hernandez Lecuona I, Garmendia Olaizola G, et al. Pseudotumor inflamatorio de vejiga. Arch Esp Urol 1999;52(4):385-387.

10. Young RH, Scully RE, Rosai J. Pseudosarcomatous lesions of the urinaryu bladder, prostate gland and urethra: a report of three cases and review of the literature. Arch Path Lab Med 1987;111:354.

11. Horn LC, Reuter S, Biesold M. Inflammatory pseudotumor of the ureter and urinary bladder. Pathol Res Pract 1997; 193:607-612.

12. Abascal Junquera JA, Trilla Herrera E, Esquena Fernández S: Pseudotumor inflamatorio vesical. Actas Urol Esp 2004;28:62-64.

13. Gyftopoulus K, Serafetenidis E, Sambaziotis D, et al. Pseudomalignant spindel cell proliferation of the urinary bladder and unusual cause of voiding symptons in a young patient. Urology 2002;60(4):698.

14. Nochomovitz LE, Orentein JM. Inflammatory pseudotumo of the urinary bladder- posible relationship to nodular fasciitis. Two cases reportss, cytologic observation, and ultraestructural observations. Amer JJ Surg Path 1985;9:366.

15. Olsen S. Tumors of the kidney and urinary tract. Colour atlas and text book. Copenhagen. Munksgaard \& Philadelphia.: Saunders 1984.

16. Fadl-ElMula I, Gorunova G, Mandahl N, et al. Chromosomal abnormalities in inflammatory pseudotumor of the urinary bladder. Cancer Genetics and cytogenetics 2003; 143:169-171.

17. Selves J, Meggetto F, Brousset P, et al. Inflammatory pseudotumor of the liver. Evidence for follicular dentric reticulum cell proliferation associated with clonal Epstein-Barr virus. Am J Surg Pathol 1996;20:747-753.

18. Sonobe H, Okada Y, Sudo S, et al. Inflammatory pseudotumor of the urinary bladder with aberrant expresion of cytorekerati. Acta Cytol 1999;43:257-262.

Dr. I. Gómez García

María Tubau, 15 - portal 3 - 1ㅇ

28050 Madrid

(Trabajo recibido el 17 febrero de 2005) 